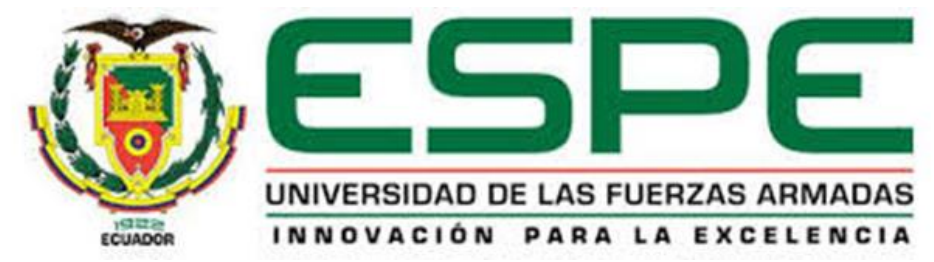

\author{
DEPARTAMENTO DE ELÉCTRICA Y ELECTRÓNICA \\ CARRERA DE INGENIERÍA EN ELECTRÓNICA E INSTRUMENTACIÓN
}
ARTÍCULO ACADÉMICO PREVIO A LA OBTENCIÓN DEL TÍULO DE INGENIERO EN ELECTRÓNICA E INSTRUMENTACIÓN

TEMA: TEACHING AND LEARNING VIRTUAL STRATEGY FOR THE NAVIGATION OF MULTIPLE-UAV

\author{
AUTORES: Rodriguez Conde Jacson Javier \\ Edison Luciano Bonilla Borja \\ DIRECTOR: Dr. Julio Francisco Acosta Núñez
}

Latacunga

Agosto, 2020 


\title{
Teaching and Learning Virtual Strategy for the Navigation of Multiple-UAV
}

\author{
Edison L. Bonilla, Jacson J. Rodriguez, Julio F. Acosta, and \\ Víctor H. Andaluz \\ Universidad de las Fuerzas Armadas ESPE \\ Sangolquí - Ecuador \\ \{elbonilla, jjrodriguez9, jfacosta, vhandaluz1\}@espe.edu.ec
}

\begin{abstract}
This paper presents a virtual application based on the engineering area teaching-learning process for the mobile robots cooperative control. The application development is basically focus on advanced control algorithms implementation in a 3D virtual environment shown in the Unity 3D graphic engine, which exchanges information in real time using mathematical software improving the control strategy. This strategy considers the multiple UAVs autonomous navigation in order to perform cooperative tasks. Finally, experimental tests that show users' interaction and immersion in the virtual environment are presented while doing cooperative navigation tasks.
\end{abstract}

Keywords - education strategy; Virtual Reality; cooperative control; tasks; feedback.

\section{INTRODUCTION}

In each generation, new technologies are being developed by humanity, what the anglosajones call game changers arise: these ideas or products make a difference between the past and nowadays means they greatly facilitate the performance of an activity, optimize processes, and improve playful learning strategies, among others. A similar model has emerged with Virtual Reality since it allows a person to interact with an artificial threedimensional visual environment or another sensory environment, to improve the user experience when carrying out an activity [1,2]. It also achieves a mental immersion state that allows the user to experience a connection between the real and virtual world, this connection is given by the haptic devices that receive and send information [3]. Virtual reality applications immerse the user in different areas of skill development, e.g. medicine to perform laparoscopic surgery [4], to treat diseases such as claustrophobia [5], and even in the future doctor virtual consultations could be performed [6,7]; military (Real-time military simulation system) [8-10]; industry for the relevant data presentation from processes to the choice of raw material [11,12]; entertainment as game systems [13], and mainly in education as systems that allow improving the methodological learning processes for students [14]. Therefore, the use of virtual reality to satisfy an educational system became a need that must be addressed by incorporating several current technologies such as VR and ICTs.

According to UNAM (2018), information and communication technologies (ICTs) comprise tools that enable the transmission, processing and storage of digitized information for their use. Mainly in education they allow students to obtain information from different places in the world at their fingertips with great fluency. The education system creates teaching methodologies that include the use of ICTs and virtual reality, incorporating new systematic strategies $[15,16]$. This strategy must satisfy the needs, the usefulness and the continuous improvement of education, e.g. [15] the impact of incorporating information technologies with virtual reality in a possible application for automotive engineering is analyzed, expanding the research horizon. Another area where teaching strategies have been developed is with robotics to immersive and collaborative learning support [17].

Robotics is also used in education for its continuous highly upward development and its innovative teaching resource, providing a learning method that allows students to create their own knowledge [18], technology challenge uses for teaching lies in the economy, educational institutions do not have financial resources to implement a series of robots for the student skills development, making the most suitable and needed tool to implemented a virtual reality, which allows to have a closer virtual experience to reality and number of robots is not a problem.

This paper developed a virtual reality application showed in Unity 3D graphic engine, as a tool to support the engineering area learning process that allows to emulate the flight of unmanned aerial robots commanded by a cooperative controller designed in the mathematical software which allows to determine the control mistakes that occur when performing a user designed task. The article proposal is summarized in Fig. 1.

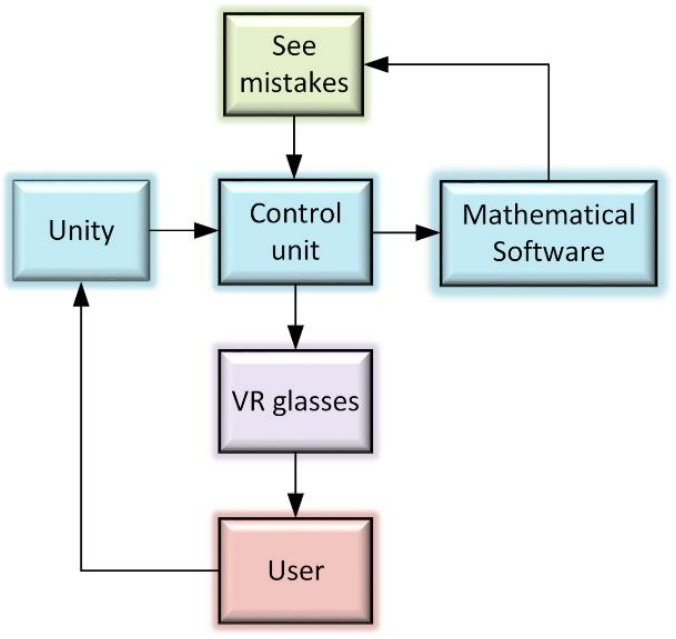

Figure 1. System architecture.

Five sections are developed in this document. Section II describes the system structure, used tools and connection levels between Unity 3D and MATLAB programs. Section III details the algorithm cooperative control for multiple UAVs. Section IV includes the results obtained. The last Section V contains the implemented application conclusions. 


\section{SYSTEM STRUCTURE}

This section describes the educational application structural scheme, from the hardware and software used to the input and output device management allowing the user to really understand the application; the scheme is divided into six stages as shown in Fig. 2.

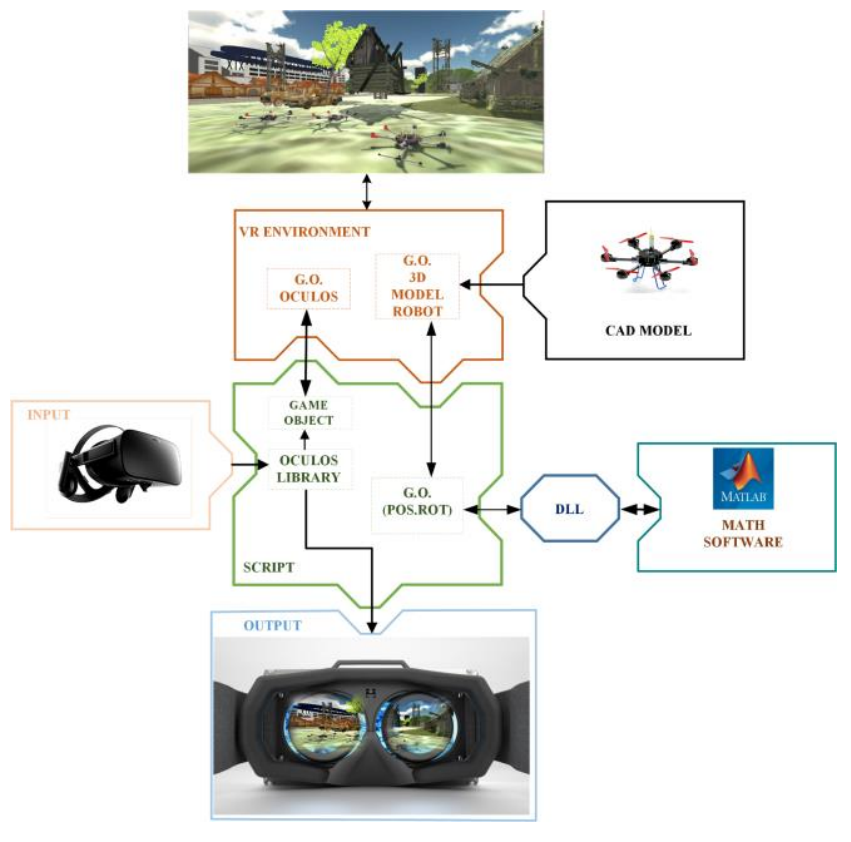

Figure 2. System structure

Virtual application development was necessary to create a VR environment, which contains 3D environment graphic interface elements including typical Unity's Assets such as buildings, trees, etc. on the other hand, the airborne robot was exported from a CAD software which allows the UAVs design their respective mechanical characteristics among the most important are propellers number, chassis, landing gear and couplings for the assembly process creating a real environment to emulate multiple in the UAVs flight. Peculiarities such as gravity, weather and mode to improve the user experience in the environment are already preestablished. The graphical interface allows to observe the development that has the cooperative network of air robots during the flight and the speeds of UAVs at that time.

The Scripts contains the codes that allow the interaction or bidirectional communication between the virtual devices and the Unity 3D graphic engine; and between the software that allows the resolution of the MATLAB mathematical part and the Unity 3D graphic engine. The bidirectional communication is developed based on the DLL (dynamiclink library) protocol that allows to obtain data of the variables that change during the UAVs flight within the virtual environment. The relevant variables that will be analyzed are speed, orientations and air robot's location at the time.

The Math software has control algorithms to control errors during the UAVs flight which receives the data sent by the graphic engine to make calculations and determine a new signal of position, orientation and speed for the UAVs, these signals are sent to the graphic engine making the aerial robots change their conditions obtaining this way an application that evolves in the time, in addition it allows to obtain graphs of errors making the difference of the wished position and the own position of the point of interest.
The Input consists on virtual devices to visualize the virtual environment according to the user position. Output achieves a mental state immersion that allows the user to get a connection with the virtual environment, deploying a friendly simulation environment for UAVs flight.

\section{Cooperative Control SCHEME}

This section describes the control structure for the application development in order to simulate multiple UAVs flight within the immersive and interactive virtual environment; it also allows to identify and control errors when performing a task designated by the user. The system consists of two subsystems as shown in Fig.3.

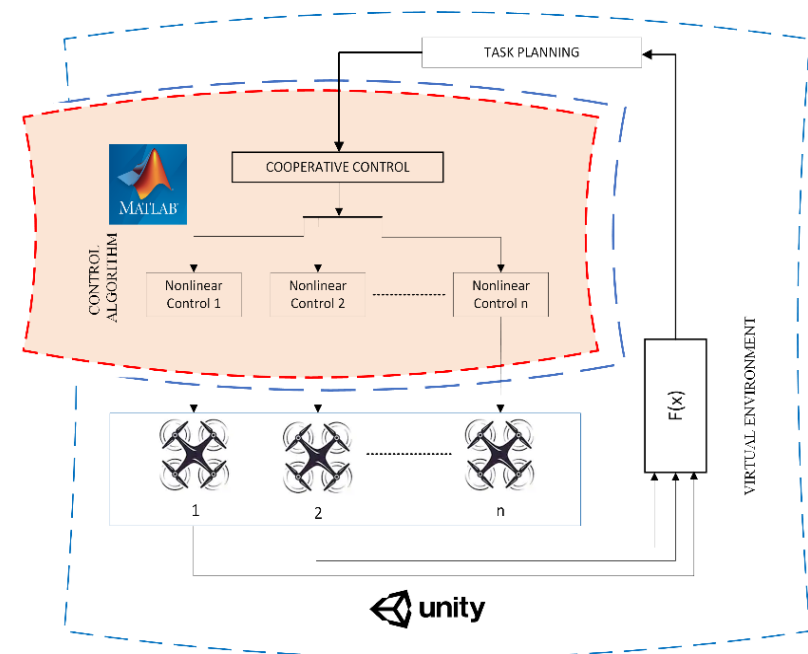

Figure 3. Multilayer training control scheme

Taking into account the subsystems functions, the application can be divided into two main subsystems: $i$ ) Virtual Environment-. It determines the user's tasks to be executed by the multiple UAVs in a cooperative job, which delimits the number of airborne robots, the initial conditions of position and orientation of the UAVs, the training scheme that the UAVs will acquire in flight, and the speed that the UAVs must take to meet the objective proposed by the user in an autonomous manner; In addition, this subsystem considers the virtual environment in which the UAVs will operate. It should be noted that this subsystem is developed in the Unity 3D graphic engine; y ii) Control Algorithm-. On the other hand, is developed in MATLAB, mathematical software that performs the reception and sending of variables (positions, orientations and speeds of the UAVs) to the Unity 3D graphic engine by means of DLLs, making the UAVs obtain movement within the virtual environment, by having access to the variables of the UAVs and the task that must be executed by the robots, the error in the positions and orientations that are produced at each instant of time is calculated by means of controllers. The controllers that were necessary to satisfy this need are: cooperative control and nonlinear control for each UAV. 


\section{Cooperative Control}

Cooperative control arises from two aerial robots' formation of which mainly interest is located in distance center that separates the UAVs forming a projection (position, orientation). To add robots to the formation it is necessary to incorporate a physical and virtual robot generating a new projection to locate the point of interest. The points projections can be seen in Fig. 4, where the performed cooperative task is being carried out by 2 robots.

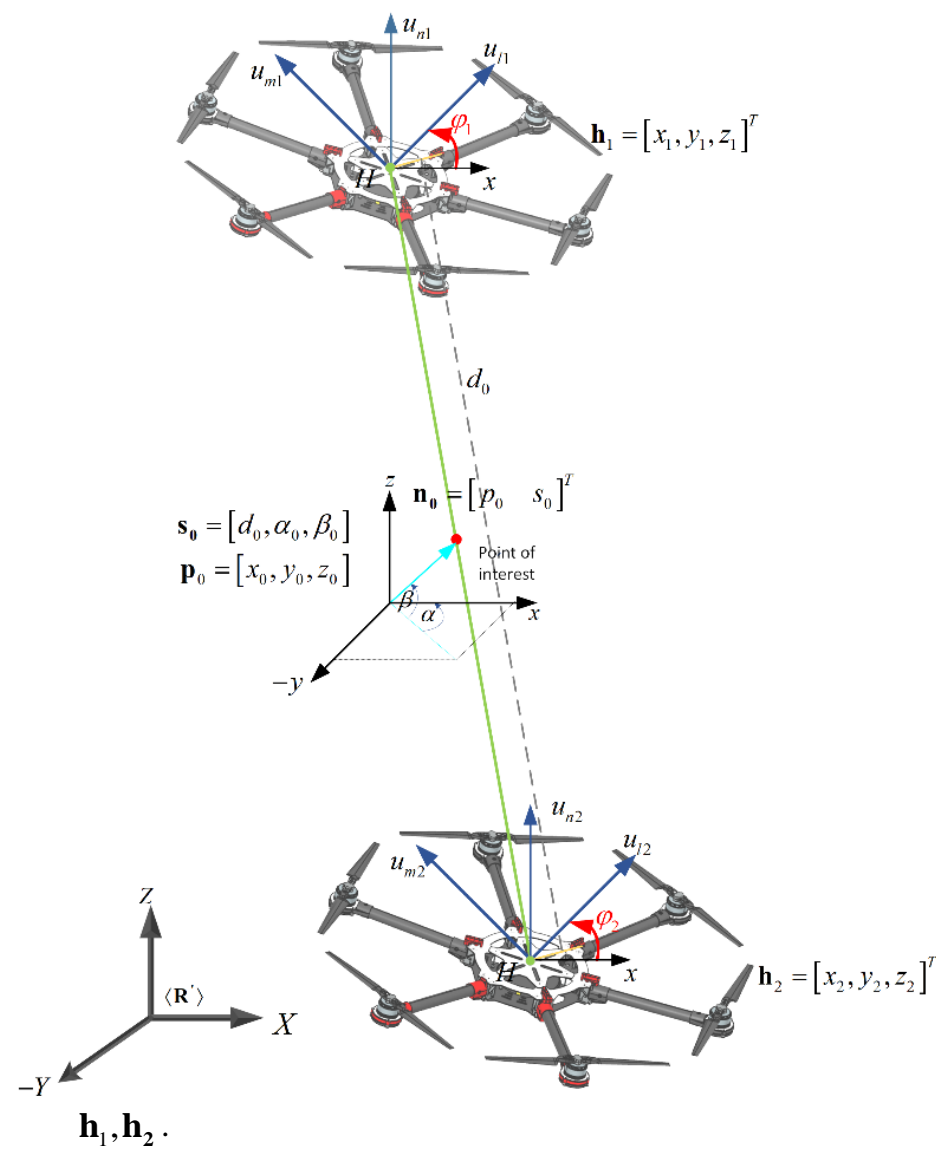

Figure 4. UAVs cooperative control diagram

The projection is between the robots position $\mathbf{h}_{\mathbf{1}}$ and $\mathbf{h}_{\mathbf{2}}$ which allow to obtain a point $\mathbf{n}_{0}=\left[\begin{array}{ll}p_{0} & s_{0}\end{array}\right]^{T}$ whose components are position $\mathbf{p}_{0}=\left[x_{0}, y_{0}, z_{0}\right]^{T}$ that establishes the midpoint between the UAVs and the $\mathbf{s}_{0}=\left[d_{0}, \alpha_{0}, \beta_{0}\right]^{T}$ where $\left(d_{0}\right)$ represents the distance between UAVs; while $\left(\alpha_{0}\right)$ y $\left(\beta_{0}\right)$ are the angles of orientation with respect to the $\mathrm{z}$-axis and $\mathrm{y}$-axis, respectively. The projection determines the point that allows to make a difference between position and orientation desired (determined by performed task) and the position and orientation that the cooperative work has at that time $\mathbf{n}_{0}$ to later correct the error and generate new speeds for the UAVs. The expressions that allow the projections to be made are denoted by:

$$
\mathbf{p}_{0}=\frac{1}{2}\left[\begin{array}{lll}
\left(x_{2}+x_{1}\right) & \left(y_{2}+y_{1}\right) & \left(z_{2}+z_{1}\right)
\end{array}\right]^{T}
$$

$$
\mathbf{s}_{0}=\left[\begin{array}{c}
\sqrt{\left(x_{2}-x_{1}\right)^{2}+\left(y_{2}-y_{1}\right)^{2}+\left(z_{2}-z_{1}\right)^{2}} \\
\tan ^{-1}\left(\frac{z_{2}-z_{1}}{x_{2}-x_{1}}\right) \\
\tan ^{-1}\left(\frac{y_{2}-y_{1}}{x_{2}-x_{1}}\right)
\end{array}\right]
$$

where $x_{1}, y_{1}, z_{1}$ represent the first airborne robot components position; while $x_{2}, y_{2}, z_{2}$ represent the second airborne robot components position projection.

For the scalability process, a virtual robot is added (the point obtained from a projection of two robots that has a position component and an orientation component) for each physical robot, since the projections are made by pairs of robots. In Fig. 5 it is proposed that the cooperative task is performed by 3 robots, as initial projection of $\mathbf{h}_{1} y \mathbf{h}_{2}$ you get a virtual robot $\mathbf{h}_{\mathbf{v}}$ the same as the one with position $\mathbf{p}_{v}=\left[x_{v}, y_{v}, z_{v}\right]^{T}$ and guidance $\mathbf{s}_{v}=\left[d_{v}, \alpha_{v}, \beta_{v}\right]^{T}$ which allow the formation of the two UAVs to be fixed with respect to the interest point of. The following projection is made by $\mathbf{h}_{3} \mathrm{y} \mathbf{h}_{v}$ which allow to obtain a point $\mathbf{n}_{0}=\left[\begin{array}{ll}p_{0} & s_{0}\end{array}\right]^{T}$ the components of which are $\mathbf{p}_{0}=\left[x_{0}, y_{0}, z_{0}\right]^{T}$ and $\mathbf{s}_{0}=\left[d_{0}, \alpha_{0}, \beta_{0}\right]^{T}$ described above. This last point allows you to set the 3 robots formation to perform the cooperative task by making the difference between the desired and the actual. For the following projections, equation (1) and (2) are used with a pair of UAVs. In Fig. 5 we can see $\mathbf{h}_{i}$ where is the $i$-th UAV that can be placed in the formation, performing the process described above.

Taking the time derivative of the forward and the inverse kinematics transformations we can obtain the relationship between the time variations of $\dot{\mathbf{h}}(t)$ and $\dot{\mathbf{n}}(t)$, represented by the Jacobian matrix $\mathbf{J}$, which is given by

$$
\dot{\mathbf{n}}=\mathbf{J}(\mathbf{h}) \dot{\mathbf{h}}
$$

and in the inverse way is given by

$$
\dot{\mathbf{h}}=\mathbf{J}^{-1}(\mathbf{n}) \dot{\mathbf{n}}
$$




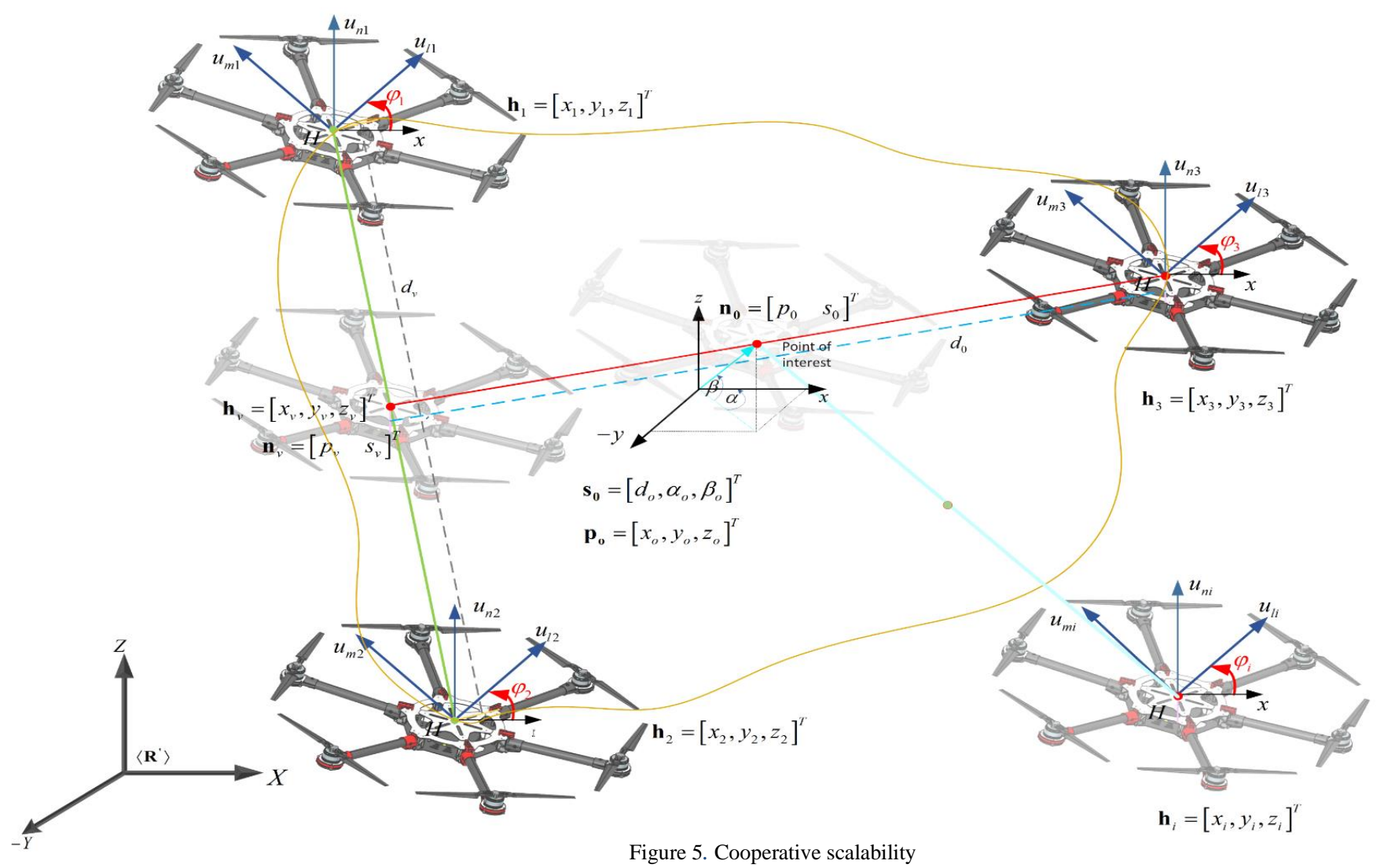

\section{Formation Controller}

The top layer in Fig. 3 sends the desired shape and position of the formation $\mathbf{n}_{d}=\left[\begin{array}{ll}p_{d} & s_{d}\end{array}\right]^{T}$ and its desired variations. $\dot{\mathbf{n}}_{d}=\left[\begin{array}{ll}\dot{p}_{d} & \dot{s}_{d}\end{array}\right]^{T}$. The formation error is defined $\tilde{\mathbf{n}}=\mathbf{n}_{d}-\mathbf{n}$ as taking the first derivative of time the following expression is obtained $\dot{\tilde{\mathbf{n}}}=\dot{\mathbf{n}}_{d}-\dot{\mathbf{n}}$. Now, by defining $\tilde{\mathbf{n}}(t)=0$ as a control objective (a system balance point), To demonstrate stability, a Lyapunov-based controller is proposed. Defining a positively defined candidate function as, $\mathbf{V}(\tilde{\mathbf{n}})=\frac{1}{2} \tilde{\mathbf{n}}^{T} \tilde{\mathbf{n}}>0$. Considering the first derivative $\dot{\mathbf{V}}(\tilde{\mathbf{n}})=\tilde{\mathbf{n}}^{T} \dot{\tilde{\mathbf{n}}}$ it is replaced $\dot{\tilde{\mathbf{n}}}=\dot{\mathbf{n}}_{d}-\dot{\mathbf{n}}$ and considered $\quad \dot{\mathbf{n}}=\mathbf{J} \dot{\mathbf{h}} \quad, \quad$ is obtained $\dot{\mathbf{V}}(\tilde{\mathbf{n}})=\tilde{\mathbf{n}}^{T} \dot{\tilde{\mathbf{n}}}=\tilde{\mathbf{n}}^{T}\left(\dot{\tilde{\mathbf{n}}}_{d}-\mathbf{J} \dot{\mathbf{h}}\right)$. In consequence, the proposed formation control law is defined as,

$$
\dot{\mathbf{h}}(t)=\mathbf{J}^{-1}\left(\dot{\mathbf{n}}_{d}+\mathbf{K} \tanh (\tilde{\mathbf{n}})\right)=\mathbf{J}^{-1} \dot{\mathbf{n}}
$$

where, $\mathbf{K}$ it's a diagonal matrix of positive gain. Introducing (3) in the time derivative of $\dot{\mathbf{V}}(\tilde{\mathbf{n}})$ is obtained,

$$
\dot{\mathbf{V}}(\tilde{\mathbf{n}})=-\tilde{\mathbf{n}}^{T} \mathbf{K} \tanh (\tilde{\mathbf{n}})<0 .
$$

As described, the equilibrium point is asymptotically stable, i.e. $\tilde{\mathbf{n}}(t) \rightarrow 0$ asymptotically.

Equation (5) represents the desired velocity vector for each UAV, relaxing the assumption of perfect speed following is considered a difference $\delta_{\dot{\tilde{n}}}(t)$ between desired and actual variations as $\delta_{\dot{\tilde{\mathbf{n}}}}=\dot{\mathbf{n}}_{d}-\dot{\mathbf{n}}$ where (4) is defined as,

$$
\dot{\mathbf{V}}(\tilde{\mathbf{n}})=\tilde{\mathbf{n}}^{T} \delta_{\dot{\tilde{\mathbf{n}}}}-\tilde{\mathbf{n}}^{T} \mathbf{K} \tanh (\tilde{\mathbf{n}})
$$

A sufficient condition to $\dot{\mathbf{V}}(\tilde{\mathbf{n}})$ be defined as negative is,

$$
\left|\tilde{\mathbf{n}}^{T} \mathbf{K} \tanh (\tilde{\mathbf{n}})\right|>\left|\tilde{\mathbf{n}}^{T} \delta_{\dot{\tilde{\mathbf{n}}}}\right| .
$$

For large values of $\tilde{\mathbf{n}}$ it can be considered that: $\mathbf{K} \tanh (\tilde{\mathbf{n}}) \approx \mathbf{K}$. While $\dot{\mathbf{V}}(\tilde{\mathbf{n}})$ will be of definite negative only if $\|\mathbf{K}\|>\left\|\delta_{\dot{\mathbf{n}}}\right\|$ making errors $\tilde{\mathbf{n}}$ decrease . For small values $\tilde{\mathbf{n}}$, it can be expressed as $\mathbf{K} \tanh (\tilde{\mathbf{n}}) \approx \mathbf{K} \tilde{\mathbf{n}}$ and the (6) can be written as $\|\tilde{\mathbf{n}}\|>\left\|\delta_{\hat{\mathbf{n}}}\right\| / \lambda_{\text {min }}(k)$ which implies that the error $\tilde{\mathbf{n}}$ is delimited by,

$$
\|\tilde{\mathbf{n}}\| \leq \frac{\left\|\delta_{\dot{\mathbf{n}}}\right\|}{\zeta \lambda_{\text {min }}(k)} ; \quad \text { with } \quad 0<\zeta<1
$$

Therefore, if $\delta_{\dot{\tilde{\mathbf{n}}}}(t) \neq 0$ the training error $\tilde{\mathbf{n}}(t)$ is ultimately delimited by (7).

\section{UAV Kinematic Modeling}

This subsection presents the kinematic model of the unmanned aerial vehicle (UAV) composed of a vector containing four speeds that allow the robot displacement 
within the three-dimensional $X, Y, Z$ space with respect to the origin $\langle R\rangle$; its movement is guided by three linear speeds $u_{l}, u_{n} \mathrm{y} u_{m}$ that allow axes movements $l, m, n$ whereas the angular velocity $w$ causes the aerial robot to rotate relative to the axis $Z$.The free body diagram of the UAV is shown in Fig. 6

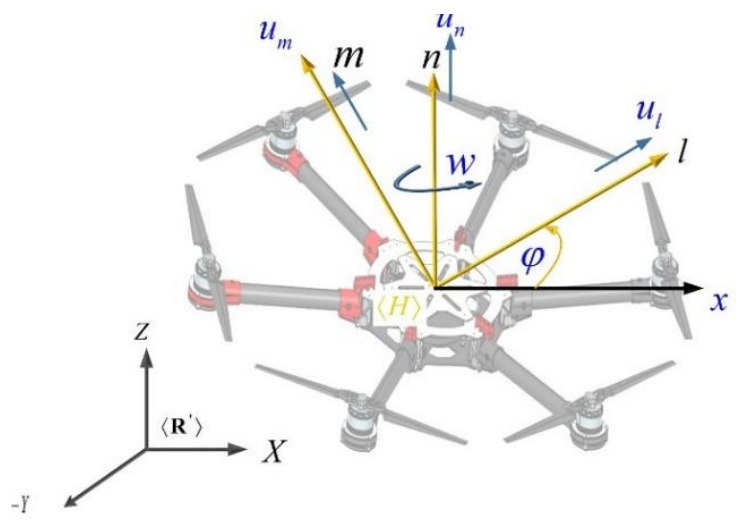

Figure 6. Schematic of the aerial robot

Each linear and angular velocity is released from the gravity center of the $\mathrm{UAV},\langle H\rangle ; u_{l}$ points to the front direction; $u_{m}$ points to the side direction $u_{n}$ vertically in the direction of the axis $Z$. The angular velocity $w$ revolves around the reference system $\langle H\rangle$ counterclockwise around the axis $Z$ (considering the top view). Therefore, the Cartesian motion of the UAV within the three-dimensional space is defined by:

$$
\begin{gathered}
{\left[\begin{array}{l}
\dot{h}_{x} \\
\dot{h}_{y} \\
\dot{h}_{z} \\
\dot{h}_{\varphi}
\end{array}\right]=\left[\begin{array}{cccc}
\cos (\varphi) & -\sin (\varphi) & 0 & 0 \\
\sin (\varphi) & \cos (\varphi) & 0 & 0 \\
0 & 0 & 1 & 0 \\
0 & 0 & 0 & 1
\end{array}\right]\left[\begin{array}{c}
u_{l} \\
u_{m} \\
u_{n} \\
w
\end{array}\right]} \\
\dot{\mathbf{h}}(t)=\boldsymbol{\Gamma}(\varphi) \mathbf{u}(t)
\end{gathered}
$$

where $\dot{\mathbf{h}}(t) \in \mathfrak{R}^{n}$ with $n=4$ which represents the velocity vector in $\{X, Y, Z\}$ and the angular velocity with respect to the $Z$; whereas $\Gamma(\varphi) \in \mathfrak{R}^{\text {nxm }}$ where $n=m$ which represents the motion characteristics of the $\mathrm{UAV}$; and the maneuverability of the UAV is denoted by the vector $\mathbf{u}(t) \in \mathfrak{R}^{n}$.

\section{Control Algorithm for the i-th UAV}

From the formation control the desired positions $\mathbf{h}_{d}=\left[\begin{array}{lll}h_{x d} & h_{y d} & h_{z d}\end{array}\right]^{T}$ of the UAV with a variation $\dot{\mathbf{h}}_{d}=\left[\begin{array}{lll}\dot{h}_{x d} & \dot{h}_{y d} & \dot{h}_{z d}\end{array}\right]^{T}$ are obtained; therefore, the control error is defined as $\tilde{\mathbf{h}}(t)=\mathbf{h}_{d}(t)-\mathbf{h}(t)$ with its variation in time $\dot{\tilde{\mathbf{h}}}=\dot{\mathbf{h}}_{d}-\dot{\mathbf{h}}$. In order to demonstrate stability, a Lyapunov-based controller is proposed. Proposing a positive candidate function defined as, $\mathbf{V}(\tilde{\mathbf{h}})=\frac{1}{2} \tilde{\mathbf{h}}^{T} \tilde{\mathbf{h}}>0$.

Taking the first derivative and replacing $\dot{\widetilde{\mathbf{h}}}=\dot{\mathbf{h}}_{d}-\dot{\mathbf{h}}$ and considering that $\dot{\mathbf{h}}=\boldsymbol{\Gamma} \mathbf{u}$ is obtained, $\dot{\mathbf{V}}(\tilde{\mathbf{h}})=\tilde{\mathbf{h}}^{T}\left(\tilde{\mathbf{h}}_{d}-\boldsymbol{\Gamma} \mathbf{u}\right)$ . The proposed control law for the $\mathrm{i}$-th UAV is defined as,

$$
\mathbf{u}=\boldsymbol{\Gamma}^{-1}\left(\dot{\mathbf{h}}_{d}+\mathbf{Q} \tanh (\tilde{\mathbf{h}})\right)
$$

where, $\mathbf{Q}$ is a positive diagonal gain matrix. By introducing (9) into the time derivative of $\dot{\mathbf{V}}(\tilde{\mathbf{h}})$ it is obtained.,

$$
\dot{\mathbf{V}}(\tilde{\mathbf{h}})=-\tilde{\mathbf{h}}^{T} \mathbf{Q} \tanh (\tilde{\mathbf{h}})<0
$$

As described, the equilibrium point is asymptotically stable, i.e. $\tilde{\mathbf{h}}(t) \rightarrow 0$ asymptotically.

Similar to formation control, if you relax the assumption of perfect speed tracking you have to $\tilde{\mathbf{u}}=\mathbf{u}_{d}-\mathbf{u}$ where (10) is defined as $\dot{\mathbf{V}}(\tilde{\mathbf{h}})=\tilde{\mathbf{h}}^{T} \tilde{\mathbf{u}}-\tilde{\mathbf{h}}^{T} \mathbf{Q} \tanh (\tilde{\mathbf{h}})$. Therefore, the error $\tilde{\mathbf{h}}$ is delimited by,

$$
\|\tilde{\mathbf{h}}\| \leq \frac{\|\tilde{\mathbf{u}}\|}{\zeta \lambda_{\text {min }}(\mathrm{Q})} ; \quad \text { with } \quad 0<\zeta<1
$$

Therefore, if $\tilde{\mathbf{u}}(t) \neq 0$ the formation error $\tilde{\mathbf{h}}(t)$ is ultimately bounded by (11)

\section{RESUlTS AND ANALYSIS}

This section presents the results of the virtual application oriented to the teaching-learning process for the engineering area. In the generated application an immersive 3D virtual environment is obtained allowing students to visualize the flight of multiple UAVs designated to perform a cooperative task. Fig. 7 shows the interface developed in the graphic engine which consists of several 3D elements such as houses, trees, stadium, mountains, etc., that makes the virtual environment friendly.

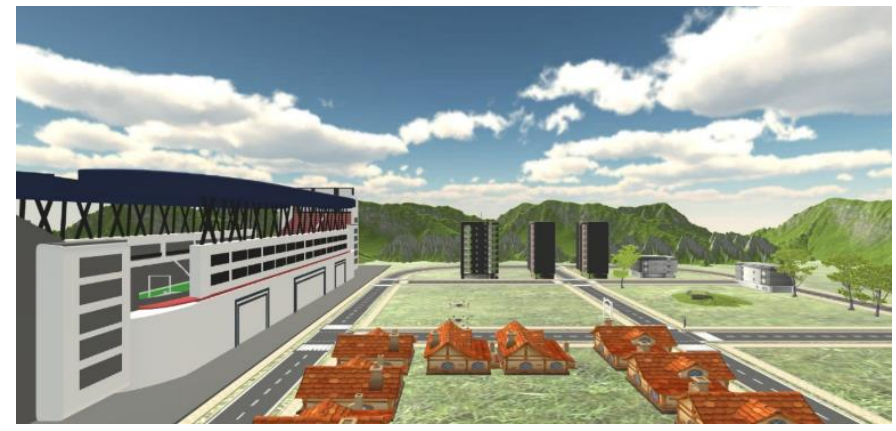

Figure 7. Schematic of the aerial robot

In order to evaluate and validate the performance of the controllers, two experimental tests were carried out. First, cooperative work was done with two UAVs, where the UAVs take off from any initial position as shown in Fig. 8, as the emulation progresses over time and the controllers perform error correction the UAVs acquire a formation pattern (Fig. 9) and maintain the point of interest in the center of the distance separating them as explained in section III. 


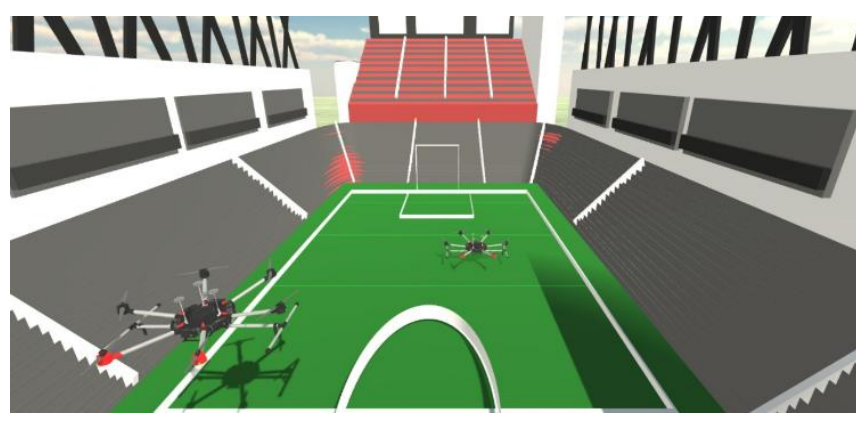

Figure 8. Cooperative work with $2 \mathrm{UAVs}, \mathrm{t}=5[\mathrm{~s}]$.

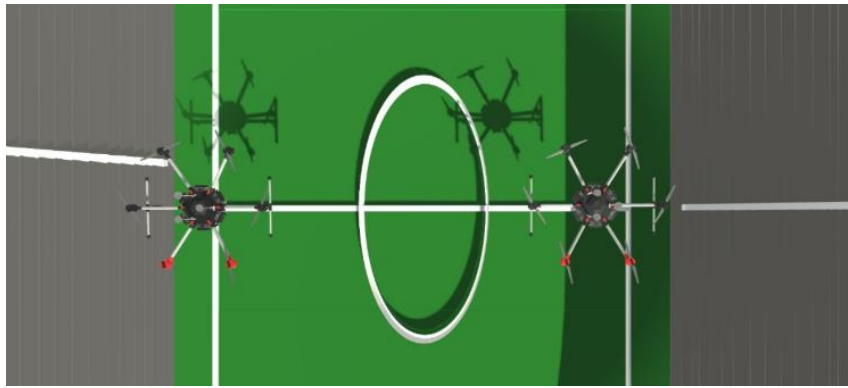

Figure 9. Cooperative work with 2 UAVs, $\mathrm{t}=10[\mathrm{~s}]$.

The graph in Fig. 10, presents the results of the experiment, it shows the desired task and the task obtained by the cooperative work showing that the controllers respond correctly and immediately.

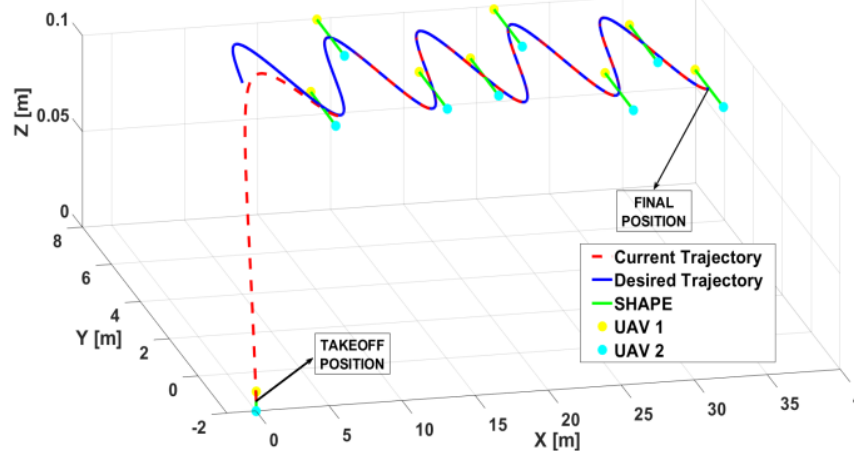

Figure 10. Training trajectory described by UAVs

The speeds generated by the controller, known as control actions, are shown in the graph of the Fig. 11,12.
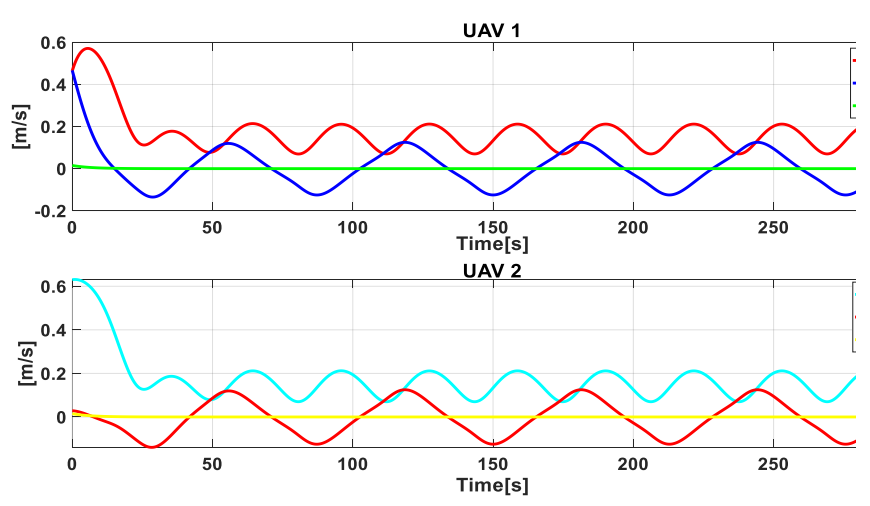

Figure 11. Linear Velocity of UAVs

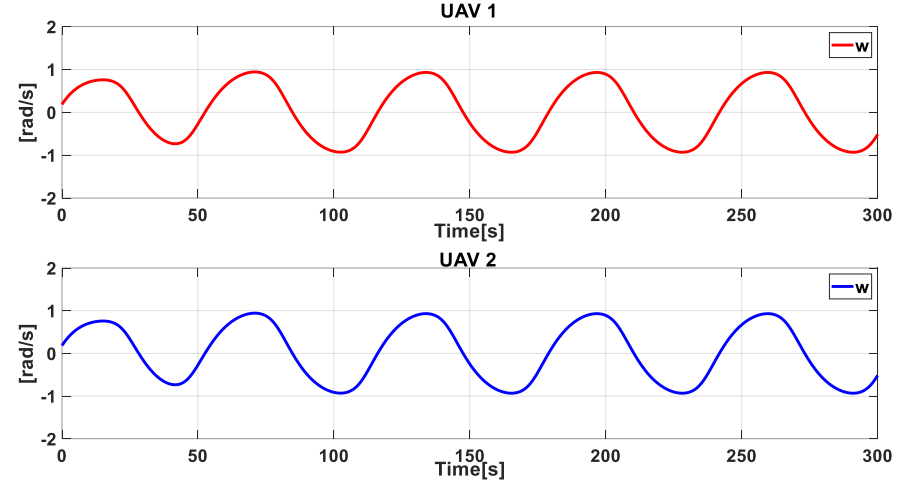

Figure 12. Angular Velocities of UAVs

For the second experiment, cooperative work was carried out with three UAVs, where the following graphs were obtained:

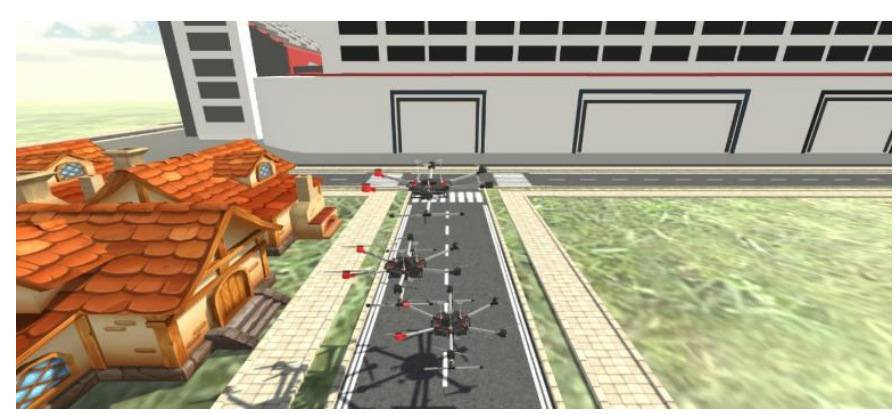

Figure 13. Cooperative work with $3 \mathrm{UAVs}, \mathrm{t}=5[\mathrm{~s}]$

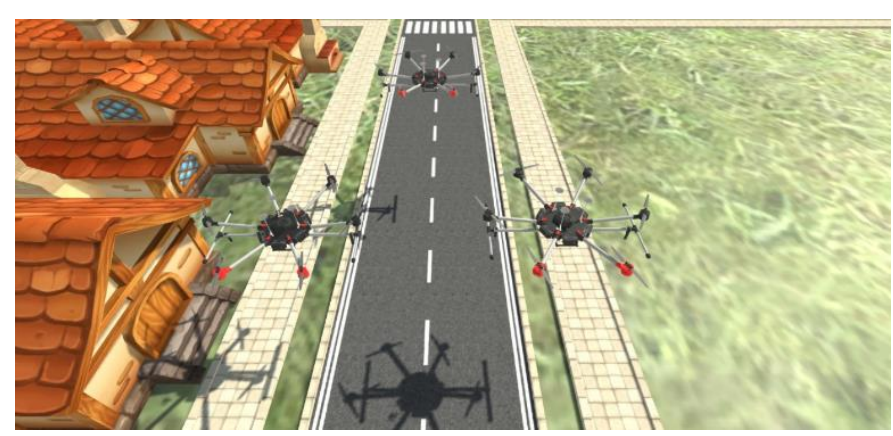

Figure 14. Cooperative work with 3 UAVs, $t=10[s]$

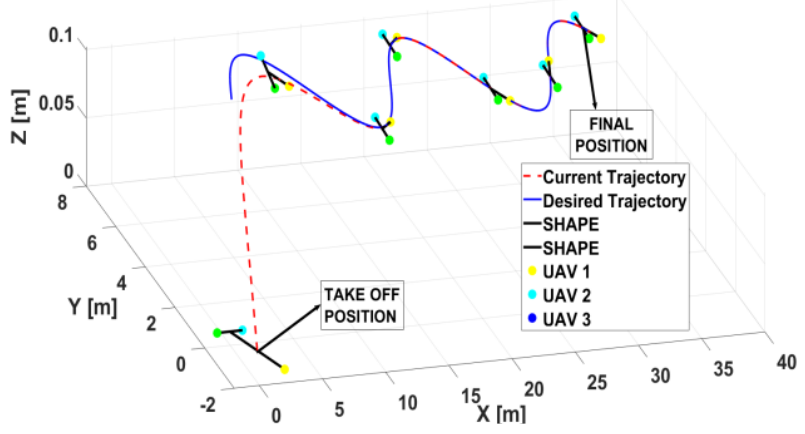

Figure 15. Training trajectory described by UAVs 

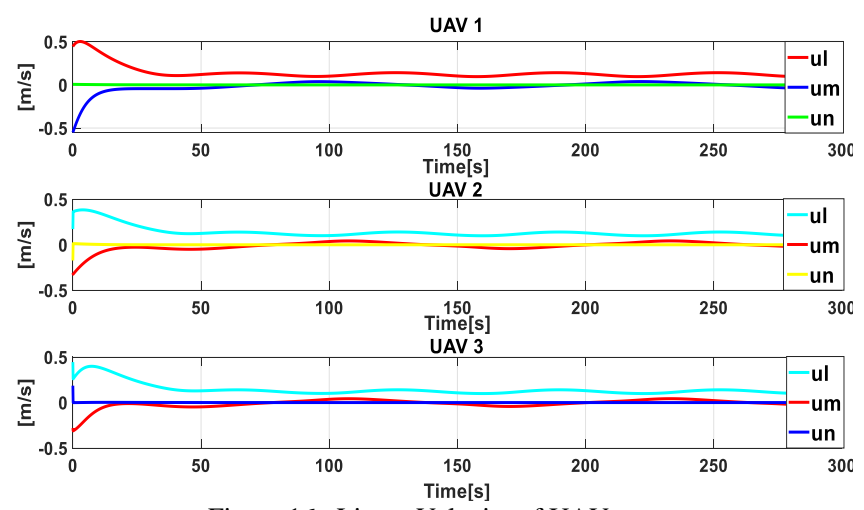

Figure 16. Linear Velocity of UAVs
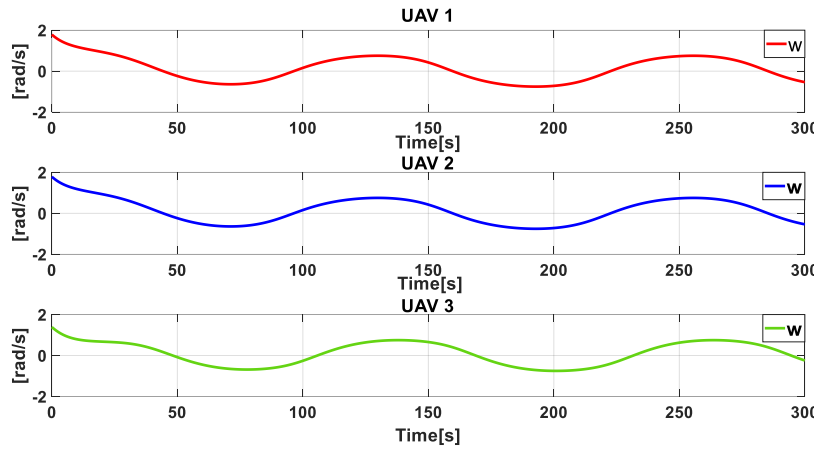

Figure 17. Angular Velocities of UAVs

\section{CONCLUSIONS}

In this work an application developed in Unity 3D platform was presented, which allows to improve the teachinglearning process for students in the engineering area while visualizing the cooperative work of multiple UAVs. Virtual Reality with the Unity 3D graphic engine presents multiple benefits such as creating a mental connection between students and the virtual environment; furthermore, it allows receiving and sending data for monitoring important variables such as position, orientation and speed of UAVs. The results of the application reflected that the developed environment is immersive and the implemented controller allows the robots to comply with the monitoring of the task designated by the user, leading to zero and asymptotic control errors both in training and in position of the UAVs.

ACKNOWLEDGMENT. The authors would like to thank the Coorporación Ecuatoriana para el Desarrollo de la Investigación y Academia- CEDIA for their contribution in innovation, through the CEPRA projects, especially the project CEPRA-XIII-2019-08 "Collaborative system of air robots for handling loads with optimal resource consumption"; also the Universidad de las Fuerzas Armadas ESPE and the Research Group in ARSI, for the support for the development of this work.

\section{REFERENCES}

[1] "Virtual reality - Education and training", Encyclopedia Britannica, 2020.[Online].Available:https://www.britannica.com/technology/vi rtual-reality/Education-and-training.
[2] Witmer, B. G., \& Singer, M. J. Measuring Presence in Virtual Environments: A Presence Questionnaire. Presence: Teleoperators and Virtual Environments, 7(3), 1998, pp.225-240.

[3] Carruth, D. W. Virtual reality for education and workforce training. 15th International Conference on Emerging eLearning Technologies and Applications (ICETA), 2017.

[4] T. Huber, M. Paschold, C. Hansen, T. Wunderling, H. Lang, W. Kneist,New Dimensions in Surgical Training: Immersive Virtual Reality La-paroscopic Simulation Exhilarates Surgical Staff,Surgical Endoscopy,2017.

[5] Yeh, S.-C., Li, Y.-Y., Zhou, C., Chiu, P.-H., \& Chen, J.-W. Effects of Virtual Reality and Augmented Reality on Induced Anxiety. IEEE Transactions on Neural Systems and Rehabilitation Engineering, 26(7), 2018, pp.1345-1352.

[6] Sik-Lanyi, C. Virtual reality healthcare system could be a potential future of health consultations. 2017 IEEE 30th Neumann Colloquium (NC), 2017.

[7] Mehta, V., Devraj, Chugh, H., \& Banerjee, P. Applications of Augmented Reality in Emerging Health Diagnostics: A Survey. 2018 International Conference on Automation and Computational Engineering.

[8] Ali, S. F., Azmat, S. A., Noor, A. U., Siddiqui, H., \& Noor, S. Virtual reality as a tool for physical training. 2017 First International Conference on Latest Trends in Electrical Engineering and Computing Technologies.

[9] Qiu, H., \& Chen, L. Real-Time Virtual Military Simulation System. 2009 First International Conference on Information Science and Engineering.

[10] Rathnayake, W. G. R. . P. S. Usage of Mixed Reality for Military Simulations. 2018 International Conference on Current Trends Towards Converging Technologies (ICCTCT).

[11] Sreevidya, P., Joseph, J. P., \& Riju, B. Data Presentation In Industries Using Virtual Reality. 2018 International Conference on Circuits and Systems in Digital Enterprise Technology (ICCSDET).

[12] Cervera, M., Grandon, N., Rivera, M., \& Besoain, F. Improving the selection of IQF raspberries in processing lines: a Virtual Reality approach for training and selecting personnel. 2018 IEEE Biennial Congress of Argentina (ARGENCON).

[13] Kodama, R., Koge, M., Taguchi, S., \& Kajimoto, H. COMS-VR Mobile virtual reality entertainment system using electric car and head-mounted display. 2017 IEEE Symposium on 3D User Interfaces (3DUI)

[14] Al-Azawi, R., \& Shakkah, M. S. Embedding augmented and virtual reality in educational learning method: Present and future. 2018 9th International Conference on Information and Communication Systems .

[15] Makarova, I., Khabibullin, R., Belyaev, E., \& Bogateeva, A. The application of virtual reality technologies in engineering education for the automotive industry. 2015 International Conference on Interactive Collaborative Learning (ICL).

[16] Contreras-Mendieta, J. A., Sarango-Lapo, C. P., Jara-Roa, D. I., Agila-Palacios, M. V., Guaman-Jaramillo, J. E., \& Betzabe Samaniego-Franco, J. Implementation of virtual worlds in distance studies. 2018 13th Iberian Conference on Information Systems and Technologies (CISTI)

[17] Segovia, M. V., \& Souza, A. Educational Robotics as a Motivational Tool for the English Teaching-Learning Process for Children. 2018 Latin American Robotic Symposium, 2018 Brazilian Symposium on Robotics (SBR) and 2018 Workshop on Robotics in Education (WRE), 2018.

[18] Plaza, P., Sancristobal, E., Carro, G., Castro, M., Blazquez, M., \& Garcia-Loro, F. Multiplatform Educational Robotics Course to Introduce Children in Robotics. 2018 IEEE Frontiers in Education Conference (FIE) 